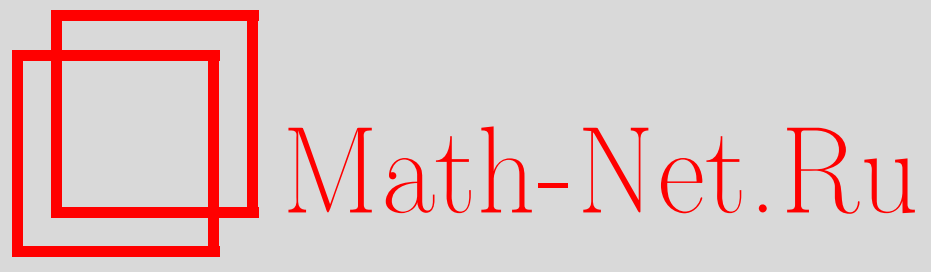

С. П. Струнков, О слабо коспектральных графах II, $M a-$ тем. заметки, 2006, том 80, выпуск 4, 627-629

DOI: https://doi.org/10.4213/mzm2855

Использование Общероссийского математического портала Math-Net.Ru подразумевает, что вы прочитали и согласны с пользовательским соглашением http://www.mathnet.ru/rus/agreement

Параметры загрузки:

IP : 54.81 .137 .203

26 апреля 2023 г., 17:23:04

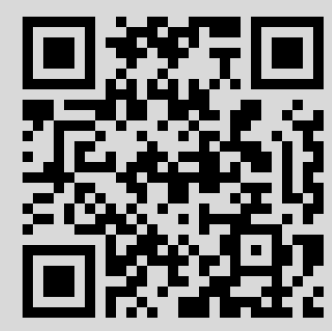




\section{О СЛАБО КОСПЕКТРАЛЬНЫХ ГРАФАХ ІІ}

\section{С. П. Струнков}

Доказывается конечность любого множества попарно неизоморфных сильно связных слабо коспектральных псевдоорграфов, множество индексов нильпотентности которых конечно.

Библиография: 5 названий.

Псевдографы или псевдоорграфы $G_{1}$ и $G_{2}[1]$ называются слабо коспектралънъми, если множества всех попарно различных характеристических чисел их матриц смежности $M_{G_{1}}$ и $M_{G_{2}}$ совпадают [2], [3]. Автором доказано в [3; следствие теоремы 1], что любое множество попарно неизоморфных слабо коспектральных связных псевдографов конечно. Для псевдоорграфов ситуация оказалась иной. В [3] в частности показано, что для любого даже сильно связного регулярного валентности $v \geqslant 2$ орграфа $G$ с вырожденной матрицей смежности $M_{G}$ существует бесконечное множество попарно неизоморфных сильно связных регулярных орграфов, слабо коспектральных с $G$. Остается открытым вопрос, обладает ли этим свойством произвольный нетривиальный сильно связный псевдоорграф с невырожденной матрицей смежности.

Индексом нильпотентности псевдоорграфа $G$ (и его матрицы смежности $M_{G}$ ) будем называть индекс нильпотентности матрицы $N$, участвующей в представлении жордановой формы $J\left(M_{G}\right)=D+N$ матрицы $M_{G}$ в виде суммы диагональной матрицы $D$ и нильпотентной $N$, т.е. наименьшее натуральное число $\mu$ такое, что $N^{\mu}=0$. Оказывается, что ограничения на абсолютные значения характеристических чисел, число попарно различных среди них и индекс нильпотентности "почти" определяют сильно связный псевдоорграф, как показывает следующая

ТЕОРема 1. Пусть $r$ - произвольное положительное число, $k$ u $\nu$ - натуральные числа, $\Sigma$ - некоторое множество попарно неизоморфных сильно связных псевдоорграфов, удовлетворяющих следующим условиям:

1) число попарно различных характеристических чисел матрицы смежности $M_{G}$ каждого псевдоорграфа $G \in \Sigma$ равно $k$, и при этом абсолютное значение каждого из них не превосходит $r$;

2) индекс нильпотентности любого псевдоорграфа $G \in \Sigma$ не превосходит $\nu$. Тогда множество $\Sigma$ конечно.

Работа поддержана Российским фондом фундаментальных исследований, грант № 05-01-01018, и ГУ-ВШЭ (договор о НИР № 511/10-05).

(C) С. П. Струнков, 2006 
ДокАЗАТЕЛЬСтво. Пусть $\lambda_{1}, \lambda_{2}, \ldots, \lambda_{k}$ - множество попарно различных характеристических чисел матрицы $M_{G}=\left(a_{i j}\right)$ некоторого сильно связного псевдоорграфа $G$, имеющего $n>1$ вершин. Так как матрица смежности $M_{G}$ сильно связного псевдооргафа $G$ неразложима $[4 ;$ п. 5.2.6] и неотрицательна, то в силу теоремы Фробениуса [5; глава 13] можно считать, что число $\lambda_{1}$ - простой вещественный положительный корень характеристического уравнения матрицы $M_{G}$ и при этом $\left|\lambda_{i}\right| \leqslant \lambda_{1} \leqslant r$ для $i=2, \ldots, k$. Это число $\lambda_{1}$ называется максимальным. Обозначим через $m$ степень минимального многочлена рассматриваемой матрицы $M_{G}$. Используя жорданову форму матрицы $M_{G}$, легко увидеть, что

$$
P(\lambda)=\left(\left(\lambda-\lambda_{1}\right)\left(\lambda-\lambda_{2}\right) \cdots\left(\lambda-\lambda_{k}\right)\right)^{\nu}
$$

является аннулирующим многочленом матрицы $M_{G}$ (т.е. $\left.P\left(M_{G}\right)=0\right)$. Следовательно, $k^{\nu} \geqslant m$.

Так как $G$ - сильно связный псевдоорграф, то для любых индексов $i, j \in\{1,2$, $\ldots, n\}$ найдется такое натуральное число $\delta$, для которого $(i, j)$-й элемент матрицы $M_{G}^{\delta}$ отличен от нуля. Минимальное среди всех таких чисел $\delta$ для индексов $i, j$ обозначим через $\delta^{i j}$, а максимальное из всех чисел $\delta^{i j}$ обозначим через $d$. Число $d$ называется диаметром псевдоорграфа $G$.

Пусть $p(t)=t^{m}+\alpha_{1} t^{m-1}+\cdots+\alpha_{m}$ - минимальный многочлен матрицы $M_{G}$. Если $d \geqslant m$, то

$$
\begin{aligned}
M_{G}^{d-m} p\left(M_{G}\right) & =M_{G}^{d-m}\left(M_{G}^{m}+\alpha_{1} M_{G}^{m-1}+\cdots+\alpha_{m} E\right) \\
& =M_{G}^{d}+\alpha_{1} M_{G}^{d-1}+\cdots+\alpha_{m} M_{G}^{d-m}=0 .
\end{aligned}
$$

Но последнее равенство невозможно, так как $(i, j)$-й элемент в левой части этого равенства, для которого $\delta^{i j}=d$, не равен нулю. Следовательно, $k^{\nu} \geqslant m>d$.

Рассмотрим матрицу

$$
B=E+M_{G}+M_{G}^{2}+\cdots+M_{G}^{k^{\nu}} .
$$

Так как $k^{\nu}>d$, то все элементы матрицы $B$ являются целыми положительными числами, и поэтому сумма элементов любой строки матрицы $B$ не может быть меньшей, чем ее порядок $n$. Легко показать, что максимальное характеристическое число матрицы $B$

$$
\Lambda_{1}=1+\lambda_{1}+\lambda_{1}^{2}+\cdots+\lambda_{1}^{k^{\nu}}
$$

где $\lambda_{1}$ - максимальное характеристическое число матрицы $M_{G}[4 ;$ п. 5.5.5]. При этом

$$
\Lambda_{1} \leqslant R=1+r+r^{2}+\cdots+r^{k^{\nu}} .
$$

Матрица $B$ содержит строку, сумма $s$ элементов которой удовлетворяет неравенству $s \leqslant \Lambda_{1}$ [4; п. 3.1.1]. Следовательно,

$$
n \leqslant s \leqslant \Lambda_{1} \leqslant R=1+r+r^{2}+\cdots+r^{k^{\nu}} .
$$

Тем самым показано, что множество чисел вершин рассматриваемого множества псевдоорграфов $\Sigma$ конечно.

Осталось показать, что число псевдоорграфов в рассматриваемом множестве $\Sigma$, имеющих заданное число $n$ вершин, конечно. Предположим, что это не так. Тогда 
$\Sigma$ содержит какой-нибудь псевдоорграф $G$ с $n$ вершинами, в матрице смежности $M_{G}=\left(a_{i j}\right)$ которого некоторый элемент $a_{p q} \geqslant 2 R^{2}+1$, где $R=1+r+r^{2}+\cdots+r^{k^{\nu}}$. Так как матрица $M_{G} \geqslant 0$ и неразложима, то любое характеристическое число $\lambda^{\prime}$ любой ее главной подматрицы удовлетворяет неравенству $\left|\lambda^{\prime}\right| \leqslant r$ [5; глава 13], [4; п. 5.5.6]. По этой причине все диагональные элементы $a_{i i}$ матрицы $M_{G}$ удовлетворяют неравенствам $0 \leqslant a_{i i} \leqslant r$. По этой же причине любой диагональный элемент $b_{i i}$ матрицы

$$
B=\left(b_{i j}\right)=E+M_{G}+M_{G}^{2}+\cdots+M_{G}^{k^{\nu}}
$$

удовлетворяет неравенствам

$$
0<b_{i i} \leqslant R=1+r+r^{2}+\cdots+r^{k^{\nu}} .
$$

Так как в матрице $M_{G}$ элемент $a_{p q} \geqslant 2 R^{2}+1$, то этот элемент не является диагональным. Рассмотрим главную подматрицу второго порядка матрицы $B$

$$
B^{\prime}=\left(\begin{array}{ll}
b_{p p} & b_{p q} \\
b_{q p} & b_{q q}
\end{array}\right) .
$$

Пусть $\lambda_{1}^{\prime}, \lambda_{2}^{\prime}$ - характеристические числа этой подматрицы. Тогда, как отмечалось выше, $\left|\lambda_{i}^{\prime}\right| \leqslant R$ для $i=1,2$. Следовательно, $\left|\lambda_{1}^{\prime}\right|\left|\lambda_{2}^{\prime}\right| \leqslant R^{2}$. С другой стороны,

$$
\left|\lambda_{1}^{\prime}\right|\left|\lambda_{2}^{\prime}\right|=\left|b_{p p} b_{q q}-b_{p q} b_{q p}\right| \geqslant b_{p q} b_{q p}-b_{p p} b_{q q} \geqslant 2 R^{2}+1-R^{2}=R^{2}+1,
$$

так как $B>0$ и $B \geqslant M_{G}$. Тем самым, теорема полностью доказана.

Из доказанной теоремы вытекает следующее обобщение цитированного выше утверждения о конечности любого множества попарно неизоморфных слабо коспектральных связных псевдографов.

СЛЕДСТВИЕ. Пусть $\nu$ - произвольное натуралъное число. Тогда любое множество попарно неизоморфных сильно связных слабо коспектралъных псевдоорграфов, индекс нильпотентности которых не превосходит $\nu$, конечно.

\section{СПИСОК ЦИТИРОВАННОЙ ЛИТЕРАТУРЫ}

[1] Харари Ф., Теория графов, Мир, М., 1973.

[2] Струнков С. П., "О слабо коспектральных графах", Докл. РАН, 379:3 (2001), 302-304.

[3] Струнков С.П., "О слабо коспектральных графах", Матем. заметки, 75:4 (2004), 614-623.

[4] Маркус М., Минк Х., Обзор по теории матрии, и матричных неравенств, Наука, М., 1972.

[5] Гантмахер Ф.Р., Теория матрич, Наука, М., 1966.

\section{С. П. Струнков}

Государственный университет - Высшая школа экономики E-mail: root@ssp.mccme.ru
Поступило 29.11.2005 